\title{
Sartre-Simon : de la « littérature engagée » aux "fictions critiques "
}

Dominique Viart

\section{(2) OpenEdition}

1 Journals

Édition électronique

URL : http://journals.openedition.org/ccs/572

DOI : $10.4000 /$ ccs. 572

ISSN : 2558-782X

Éditeur :

Presses universitaires de Rennes, Association des lecteurs de Claude Simon

\section{Édition imprimée}

Date de publication : 30 avril 2007

Pagination : 105-126

ISBN : 9782354120122

ISSN : 1774-9425

Référence électronique

Dominique Viart, "Sartre-Simon : de la « littérature engagée » aux « fictions critiques » », Cahiers

Claude Simon [En ligne], 3 | 2007, mis en ligne le 20 septembre 2017, consulté le 20 avril 2019. URL:

http://journals.openedition.org/ccs/572 ; DOI : 10.4000/ccs.572 


\section{Sartre-Simon : \\ de la « littérature engagée » aux « fictions critiques »}

Dominique VIART*

La question de 1' « engagement» de l'œuvre de Claude Simon m'a longtemps semblé devoir être posée à nouveaux frais. Dès mes premières lectures, j'ai été saisi par la prodigieuse dimension critique de l'œuvre, et je m'étonnais, encore étudiant, que la critique ne s'y intéressât point. Les mêmes remarques sont faites par Régis Debray dans Eloges qui explique combien la lecture, en prison, à Camiri, de La Route des Flandres l'a détourné de l'adhésion aux thèses marxistes' ${ }^{1}$. Mais on était encore dans une période de réception formaliste et la seule dimension critique alors commentée était celle qui montrait comment les romans de ce temps mettaient en accusation les formes d'écriture traditionnelles, et faisaient voler en éclats les modalités de la représentation réaliste ou de la linéarité narrative. Bref : affichaient leurs contestations formelles, justement. Aussi me suis-je réjoui lorsque la réception de l'œuvre s'est ouverte à de nouveaux questionnements : autour du référent historique et de la dimension autobiographique notamment.

* Université Lille Ill-Charles de Gaulle.

1 Régis Debray, Reloges, Gallimard, 1986. 
Les premiers commentateurs à en relever la dimension critique non réductible aux considérations formelles furent Lucien Dällenbach, dans son texte sur « la question primordiale » qui attirait l'attention sur la lettre adressée au narrateur prisonnier de La Route des Flandres par son père déplorant le bombardement de la Bibliothèque de Leipzig et sur la « prise de position philosophique, éthique et politique $»^{2}$ que ce texte constitue - mais se concentrait ensuite sur la seule question $d u$ primordial, du primal et de l'archaïque ; et, d'autre part, Francine Dugast qui mettait l'accent sur « la fonction tragifiante de l'ancêtre $»^{3}$, et sur les contestations des Lumières qu'opère cette figure. Tous deux font apparaître la forte querelle de Simon envers l'idéalisme humaniste. J'ai poursuivi ce travail dans la dernière partie d'Une mémoire inquiète ${ }^{4}$, en proposant pour décrire la pratique de Claude Simon, une troisième voie qui permettait d'échapper à la dichotomie bien connue entre un engagement de la littérature (version Sartre) et un engagement dans la littérature (version Mallarmé, ou Bataille) qui est aussi à certains égards, comme le montre Benoît Denis ${ }^{5}$, un « désengagement » de la littérature. Cette troisième voie, j'ai proposé de la nommer engagement par ou avec la littérature : elle me semble innover une pratique spécifique, que du reste la littérature contemporaine développe avec de plus en plus d'insistance: celle de la « fiction critique». Je ne reviens pas maintenant sur cette proposition, dont on peut désormais lire la présentation et les arguments, pas plus que sur l'une des modalités de son exercice, celle de la "métaphore critique », exposée à Cologne lors du colloque de $2004^{6}$.

1 Lucien Dällenbach, "La Question primordiale», Sur Claude Simon, Minuit, 1987.

2 Op. cit., p. 67

3 Francine Dugast, «Le Spectre de l'ascendance. Fonction tragifiante du personnage de l'ancêtre au fil de l'œuvre de Claude Simon ", Revue des Sciences Humaines, $\mathrm{n}^{\circ} 215,1989$.

4 Dominique Viart, Une mémoire inquiète, PUF, 1997.

5 Benoît Denis, «Engagement littéraire et morale de la littérature » in Emmanuel Bouju (dir.), L'Engagement littéraire, PU de Rennes, 2005, p. 31.

${ }^{6}$ Dominique Viart, «Une écriture nomade. La puissance critique de la métaphore simonienne ", in Irene Albers et Wolfram Nitsch (dir.) Transports. Les Métaphores de Claude Simon, Frankfurt am Main, Peter Lang, 2006. 
Je voudrais en revanche continuer l'investigation de la dimension critique de l'œuvre simonienne en apportant plusieurs éléments supplémentaires au dossier, lui-même déjà complété par les travaux de Nathalie Piégay-Gros sur la «poétique du montage » dans Le Jardin des Liantes en lien avec à la fois la critique et l'exercice de l'engagement. Évidemment, réfléchir aux éléments critiques mis en œuvre(s) par l'écriture de Simon suppose de tenir compte en même temps de ses propos hostiles à l'engagement de la littérature, tels qu'ils sont formulés dans le Discours de Stockholm et divers entretiens, notamment dans celui accordé à Bernard-Henri Lévy pour son livre Les Aventures de la liberté ${ }^{1}$. Ils viennent d'être rappelés et je ne m'y attarde pas. Mon propos traitera plus précisément de la critique sociale dans l'œuvre de Simon, et des griefs que l'on peut lui adresser du point de vue d'une position plus orthodoxe de l'engagement littéraire ; des questions connexes de l'observation, du témoignage et du compte-rendu poïétique de l'expérience ; et enfin des éléments constitutifs de l'éthique simonienne : l'importance accordée notamment à la matérialité et à une certaine forme de représentation de soi.

\section{Critique ou satire sociale ?}

Il n'y a guère que trois grandes formes esthétiques de l'engagement littéraire et de la critique socio-politique dans la littérature celle qui s'exerce dans le livre - ou la pièce - à thèse, à l'image de ces romans étudiés par Susan Suleiman sous la catégorie de l'autorité fictive $^{-}$; celle qui se déploie dans l'orbe du roman réaliste et ses divers avatars, dont le principe est celui de l'observation critique ; celle enfin qui s'inscrit dans le geste moraliste et stigmatise des comportements, qu'il s'agisse de les exposer dans des "caractères» ou dans des "pamphlets ». La première pratique repose sur un art du discours quand bien même celui-ci se développe par le truchement d'une narration et d'une diégèse exemplaire ; la seconde sur une prise en compte du réel; la troisième sur une position éthique. Bien sûr ces trois formes et pratiques ne sont pas étanches. Elles se recoupent même sou-

Bernard-Henri Lévy, Les Aventures de la liberté, Grasset, 1991, pp. 12-21.

2 Susan Suleiman, Le roman à thèse ou l'autorité fictive, PUF, 1983. 
vent. Elles ne balisent pas non plus complètement le champ, mais on peut considérer, je crois, que les autres pratiques se ramènent peu ou prou à cette partition. Le théâtre épique brechtien relève par exemple d'une mise en scène du réel encadrée de discours ; le conte philosophique d'une thèse illustrée par une histoire.

Qu'en est-il de Claude Simon ? Nous savons qu'il n'a pas de discours à défendre - il l'a souvent répété - mais consacre au contraire une part de son énergie à contester ceux qui se tiennent ici ou là, particulièrement les discours rousseauistes de l'ancêtre conventionnel, les rationalisations voltairiennes du père obèse de La Route des Flandres, la foi des révolutionnaires dans la pensée de Marx, cet « autre Moïse » (comme il le nomme dans Les Géorgiques), dont Les Corps conducteurs livrent la caricature. Même le simple discours de progrès de la Troisième république est l'objet de développements caustiques dans L'Acacia. Nous savons aussi que son observation du réel ne saurait souscrire à l'esthétique réaliste, mainte fois condamnée.

Cependant les choses peuvent, à y regarder de près, paraître plus ambivalentes. Cet écrivain qui, comme le narrateur le confie à la fin de L'Acacia, a relu tout Balzac, met en scène à sa façon une bien insistante " comédie humaine». Toute une galerie de portraits ressort des romans : paysans, militaires - gradés ou non -, aristocratie désargentée, mondanités de province, juifs du sentier, gitans des marches d'Espagne, domestiques, ecclésiastiques, universitaires, tribuns politiques, peintres, poètes pédérastes... et j'en oublie. Les lieux et les milieux sont aussi objets de descriptions : cafés, ateliers, bordels, salons artistes ou aristocrates, plage mondaine, champs de course, habitats divers sont visités. Certes jamais avec l'insistance volontiers exhaustive du réalisme, et sans souscrire non plus à ce que Philippe Hamon a appelé le "discours contraint». L'accent est plutôt mis du reste sur des lieux de passage et de brassage : gares, trains, hôpitaux, places publiques... et ces lieux ne sont jamais qu'esquissés mais souvent la phrase qui les dit, déploie une réflexion sur les us et coutumes, les modes d'être qui s'y affichent. Simon insiste ainsi sur quelques conduites et comportements qui témoignent d'une im- 
prégnation culturelle et sociale : les salons mondains de Perpignan non nommée, les usages des propriétaires terriens dans les lettres de L.S.M. à Batti, les Juifs qui célèbrent le Yom Kippour dans un camp de prisonniers. Et l'écrivain ne recule pas devant la caricature, avec Wack, par exemple, qui incarne dans $\mathrm{Fa}$ Route des Flandres, le paysan obtus dont le nom « Wack» signifie « vache » en flamand.

En fait, le texte procède volontiers par brèves saynètes, par aperçus que le narrateur n'entoure pas d'une glose « omnisciente». Mais il manifeste à l'endroit de ces saynètes une pulsion discursive qui multiplie les commentaires, les analogies, les rapprochements et finit par construire ainsi un point de vue critique. Certes celui-ci n'est jamais exposé comme une " thèse »: il relève toujours de l'hypothèse, $\mathrm{du}$ « comme si... ». Est-ce à dire que le regard serait celui du moraliste ? Peut-être, mais alors celui d'un moralisme paradoxal, tout en fragments et en déplacements, inséré dans des dérives discursivoimaginatives, un moralisme du constat, sans véritables valeurs affichées, assez distant et dont l'axiologie ne se donnerait qu'en creux et de manière souvent indécidable. Si l'on s'emploie cependant à en décrypter les éléments, on y découvre une conception de l'homme essentiellement régi par ses besoins primaires et ses pulsions, où dominent l'appétit du pouvoir, les dimensions sexuelle et excrémentielle. La société qui en découle apparaît comme un monde de la frustration, du désir borné, des ambitions fausses, du souci de paraître, des fallacieuses légitimations et des pièges discursifs.

En contrepoint de ces constats, le texte ne propose guère de valeurs positives. Pas même, par exemple, l'abnégation des deux sœurs dans L'Acacia, victimes autant que saintes laïques du dévouement familial. Aussi peut-on être tenté d'y voir une version noire du moralisme, celle d'un Céline auquel Simon emprunte son imaginaire parfois scatologique et même, ici et là, quelques formules et sarcasmes («casse pipe», «écrire sa page d'Histoire »...). La représentation de l'Histoire en «vieille ogresse » à « l'anus ridé », celle de l'homme en le "gorillus sapiens" qui trône au faîte de ses immondices, la révolution comparée dans Le Palace à un fœtus pourrissant en sont d'autres exemples. 
On rencontre là la première objection qui peut être adressée à l'idée d'une forme simonienne de l'engagement avec la littérature : celle d'une critique certes, mais qui demeurerait anarchique et non organisée.

Nathalie Piégay-Gros rapporte ainsi la tonalité du discours de Simon à la satire. Son " évitement du point de vue idéologique », écritelle, "dénude les vices et les tares des institutions et des individus", sans les «prendre dans la trame d'une dialectique ${ }^{1}$. À quoi l'on pourrait d'ailleurs objecter que la satire n'est pas indemne d'idéologie : les œuvres de Céline en administrent fortement la preuve. Mais surtout, il ne me semble pas que l'exercice simonien de la «fonction idéologique de la narration », pour employer la terminologie de Jakobson, relève de la satire. Il y va d'une différence de style et d'enjeux. La proximité avec Céline s'arrête à une parenté de motifs, d'expression et d'images. Contrairement à ce que l'on pourrait croire - et contrairement à ce que Bernard-Henri Lévy a voulu nous faire croire en faussant la transcription de son entretien avec Simon -, ce ne sont pas non plus des raisons éthiques qui distinguent ces deux écrivains l'un de l'autre : «Je n'ai jamais été un grand admirateur de Céline », dit Simon à BHL. "Mais pas pour des raisons morales. Le genre "imprécateur" ne me séduit guère. $»^{2}$ (BHL a « oublié » de transcrire la négation « pas » dans son texte) $)^{3}$.

La contestation de Céline par Simon est bien plus profonde que celle que lui attribue BHL en trichant avec le propos de Simon : si elle reconnaît le talent descriptif ${ }^{4}$, elle conteste le "style impréca-

1 Nathalie Piégay-Gros, « Mélancolie du montage. Le Jardin des Liantes de Claude Simon », in Emmanuel Bouju (dir.), L'Engagement littéraire, PU de Rennes, 2005, p. 286.

2 Bernard-Henri Lévy, op. cit., p. 18.

3 Cf. Claude Simon, "L'inlassable réancrage du vécu », Entretien avec Mireille Calle-Gruber (1993), in Mireille Calle-Gruber, Le Grand Temps. Essai sur l'œuvre de Claude Simon, Lille, Presses Universitaires du Septentrion, 2004, p. 250.

4 «Les meilleurs de ses livres sont D'un Château l'autre et Nord. Comme s'il avait été sauvé par son indignité même : la description de Berlin bombardé avec les balcons qui pendent comme des festons de dentelle, la traversée dans un train glacé de l'Allemagne en ruine, les dernières pages : c'est superbe... ! » \{in BernardHenri Lévy, Les Aventures de la liberté, op. cit.). 
teur ». C'est-à-dire le style, exacerbé, de la satire. Or la démonstration de ce rejet est faite très tôt par Claude Simon, dès Le Vent dont l'incipit replie la posture célinienne sur une conception balzacienne de la représentation et lui oppose toute une réflexion sur la difficulté même de la représentation et, partant, du jugement. On se souvient de ces quelques pages : le livre commence avec le propos d'un notaire - figure balzacienne s'il en est - qui martèle son point de vue sur Montès avec les catégories de Balzac pour penser le monde et la virulence de Bardamu pour les énoncer :

«Un idiot. Voilà tout. Et rien d'autre. Et tout ce qu'on a pu raconter ou inventer, ou essayer de déduire ou d'expliquer, ça ne fait encore que confirmer ce que n'importe qui pouvait voir du premier coup d'œil. Rien qu'un simple idiot. Seulement, lui, avec le droit de se promener en liberté, de parler aux gens, de signer des actes et de déclencher des catastrophes. Parce qu'il paraît que les médecins classent les types comme ça dans les inoffensifs. Très bien. C'est leur affaire. Mais si, au lieu de se contenter de leur avis, on demandait aussi celui des gens comme nous qui en savent peut-être un peu plus long sur l'espèce humaine que tous ces types de la Faculté... Parce que, écoutez-moi : en fait de spécimens humains, tout défile ici, vous pouvez me croire, et en ce qui concerne les mobiles auxquels obéissent les gens, si j'ai appris quelque chose pendant les vingt ans que j'ai passés dans cette étude, c'est ceci : qu'il n'en existe qu'un seul et unique : l'intérêt. Et alors, voilà ce que je dis... ». ( $V, 9$ / Pléiade 3)

Cette véritable réécriture célinienne de Balzac est suivie comme on le sait d'une longue réflexion sur la vanité de chercher les causes profondes de la réalité :

[...] un peu comme si on essayait de recoller les débris dispersés, incomplets, d'un miroir ${ }^{1}$, s'efforçant maladroitement de les réajuster, n'obtenant qu'un résultat incohérent, dérisoire, idiot, où peut-être seul notre esprit, ou plutôt notre orgueil, nous enjoint sous peine de folie et en dépit de toute évidence de trouver à tout prix une suite logique de causes

\footnotetext{
Celui du réalisme, bien sûr.
} 
et d'effets là où tout ce que la raison parvient à voir, c'est cette errance, nous mêmes ballottés de droite et de gauche, comme un bouchon à la dérive, sans direction, sans vue, essayant seulement de surnager et souffrant, et mourant pour finir, et c'est tout... $(V, 10 /$ Pléiade 4$)$

Ce n'est donc pas « simplement» un rejet des idées à quoi nous assistons à l'orée de l'œuvre reconnue par Simon, mais à une invalidation des procédures mêmes du jugement social, lesquelles seraient, selon cet incipit, les mêmes finalement chez les réalistes-positivistes que chez les pamphlétaires-populistes. On aura bien sûr remarqué la reprise et le glissement du terme « idiot» qui ne qualifie plus l'homme évoqué, mais le résultat de la réflexion qui tente de s'y porter.

La même critique frappe donc de concert le logos des réalistes et la logorrhée des pamphlétaires, tous trop sûrs de leur fait. Cette critique dispose que la connaissance est forcément fragmentaire et se recompose plus ou moins bien comme un puzzle jamais correctement agencé. Elle montre sur pièces qu'ipsofacto les discours qui paraissent les mieux tenus, les plus maîtrisés, relèvent eux-mêmes du passage en force. Simon démontre par la puissance critique de ses textes que, comme l'écrit Marc Angenot : «Les grandes idéologiesdoctrines mêmes, qui peuvent sembler former les secteurs les plus "solides" du discours social et dont la systémacité s'oppose le plus nettement à la textualité littéraire, ne sont que des bricolages sur du déjà-là idéologique qu'elles refaçonnent $[\ldots] »{ }^{1}$. Or, l'écriture simonienne s'emploie à défaire ce bricolage, à en afficher les raccords et les discordances. Et elle le fait en articulant ces branlants échafaudages sur les comportements individuels et sociaux. À cet égard, le travail de Claude Simon me paraît donc, mutatis mutandis, de même nature que celui accompli par Michel Foucault. Il vise aussi à élaborer une critique des « discours». Mais là où le philosophe s'en prend directement aux discours institutionnels (la justice, la psychiatrie, la médecine, etc.), par où les institutions se constituent et se légitiment 
elles-mêmes, Simon observe les comportements individuels et sociaux, les modes d'être et de relation qui incarnent ces discours implicites dans le corps social. Et il ne le fait pas à partir d'une position « philosophique » ni même « historienne" : sa position est celle d'un écrivain observateur inclus dans le champ social qu'il commente, agacé par les pratiques qu'il observe.

Un exemple est assez probant : celui des « castes ». Le terme revient plusieurs fois dans les romans de l'écrivain. Le début du Tramway démasque ainsi, par exemple, sous l'organisation sociale de la province française, un crypto système de "castes ", qui se trouvait déjà évoqué dans $\mathrm{Ta}$ Toute des Flandres, à la faveur du glissement paronomastique de la «lignée, la race, la caste, la dynastie des de Reixach » $(R F, 49$ / Pléiade 227). Semblablement Thérèse, la bonne du Tramway, ne fait pas partie "d'une classe sociale différente mais d'une sorte de caste, d'intouchables». Et le wattman est « un personnage à la fois assez misérable, d'une caste inférieure, condamné à une muette solitude " (Tram., 12). Comme l'a bien montré Vincent Joss, le texte apparente ces personnages aux sutra, ces intouchables indiens de la plus basse caste sociale, et fait apparaître leur « fonction comme la valeur d'usage de certains êtres dans une société hiérarchisée ${ }^{1}$. L'expression « valeur d'usage » est du reste utilisée par Simon à propos de Madame Espinosa (Tram., 136) et souligne la réification à l'œuvre dans la relation sociale. Au delà de la simple observation donc, une réflexion s'impose en filigrane : elle montre le cloisonnement qui régit le corps social. Simon retrouve ainsi à sa manière :

Chacune des catégories sociales détermine la conduite des membres qu'elle comprend, elle leur impose des motifs d'action bien définis ; elle leur imprime sa marque, une marque propre et bien distincte pour chaque groupe, avec une telle force que les hommes faisant partie des classes sociales séparées, bien qu'ils vivent dans un même milieu et à la même époque, nous donnent parfois l'impression qu'ils appartiennent à des espèces différentes ${ }^{2}$.

1 Vincent Joss, «La critique sociale dans Le Tramway de Claude Simon », Roman 20-50 $\mathrm{n}^{\circ} 37$, juin 2004.

2 Maurice Halbwachs, Esquisse d'une psychologie des classes sociales, Marcel Rivière, 1955, p. 210. 
Donc le texte, premièrement: se refuse le socle assuré d'une conviction sociale; deuxièmement : énonce que le savoir ne s'élabore jamais que comme incomplétude et en approximations ; troisièmement : dispose cependant des systèmes analogiques qui font apparaître des logiques sociales sous-jacentes - que le discours humaniste démocratique et émancipateur ne saurait reconnaître. Ce en quoi il va bien au-delà d'un simple geste satirique.

\section{L'événement du texte : témoigner vs écrire}

Mais, ce faisant, l'écrivain semble s'installer dans la position de l'observateur. Or cette position d'observateur et non d'acteur exclut ces textes du champ de la littérature engagée, laquelle suppose de l'artiste qu'il « renonce à une position de simple spectateur», lit-on dans le dictionnaire Robert. Simon donne ici prise à la critique sartrienne telle qu'elle s'énonce dans la "Présentation des Temps modernes». Cette extériorité du point de vue serait donc la seconde objection susceptible d'empêcher de reconnaître à l'œuvre de Simon une dimension « engagée». Une telle objection peut recevoir deux réponses, du reste déjà partiellement formulées ailleurs. Notons d'abord que l'acte d'écriture, quel qu'il soit, est forcément détaché de l'action elle-même: on ne saurait écrire en agissant. Marc Angenot le souligne justement à propos de l'écriture engagée elle-même : la littérature "n'est qu'un certain (et incertain) travail après coup sur le discours social et tire ses caractères du fait de venir après que tout soit déjà dit $»^{1}$.

Notons encore que cette extériorité n'est jamais, chez Simon, celle d'un «surplomb». Le point de vue que l'écrivain adopte est " immergé » et ce, doublement, ce qui en constitue l'originalité. Il l'est, d'une part - c'est le cas de Ta Route des Flandres par exemple -, dans l'événement, vécu et restitué au ras des choses. Ce qui n'est encore rien d'autre que le point de vue de Fabrice à Waterloo. Mais surtout il l'est aussi dans l'écriture qui ne se dégage pas a posteriori de cette immersion. Au contraire : elle redouble l'immersion dans l'événement par l'immersion dans le texte de «l'écriture en train de se faire ». Toutes les modalités de la phrase simonienne, sa célèbre 
" écriture au présent de l'écriture », témoignent de cette immersion, tant la phrase semble elle-même ne pas savoir où elle va ni comment elle se terminera au moment où elle commence. L'idée, la conception, même a posteriori, de l'événement ne préludent pas à la phrase qui l'écrit mais se constituent dans son mouvement-même. C'est là, du reste, le cœur des réserves que Simon peut formuler envers toute littérature engagée, laquelle conteste la position "observatrice» en soulignant que l'écrivain est « embarqué », mais se l'accorde à ellemême quand il s'agit de passer à l'écriture. L'écrivain engagé doit forcément en passer par une position du retrait. Ce qui n'est pas le cas du narrateur simonien, toujours immergé dans son texte comme dans l'événement. Ou, pour le dire autrement: hors de tout retrait, le narrateur simonien est toujours impliqué, embarqué, dans l'événement même du texte.

On achoppe ici sur une critique du témoignage. Pour Sartre, « il faut témoigner et représenter le monde $»^{1}$, sans pour autant adopter une position surplombante, extérieure au monde, ce qui n'est du reste pas sans paradoxe comme le montre Jean-François Louette en soutenant que la littérature engagée est et n'est pas une « littérature de témoignage $»^{2}$. Dans une telle réflexion, le témoignage se soutient d'une présence aux événements historiques et de leur formulation dans l'économie propre du langage que l'on ne saurait séparer. C'est l'assertion de Derrida dans Poétique et politique du témoignage, selon laquelle: "Tout témoignage engage une expérience poétique de la langue $»^{3}$. Or pour Simon cette « expérience poétique » invalide le témoignage même, comme le montrent dans Les Géorgiques les commentaires faits à propos d'Hommage à la Catalogne d'Orwell :

Peut-être espère-t-il qu'en écrivant son aventure il s'en dégagera un sens cohérent. Tout d'abord le fait qu'il va énumérer dans leur ordre chronologique des événements qui se bousculent pêle-mêle dans sa mémoire ou se présentent selon des

1 Jean-Paul Sartre,Qu'est ce que la littérature? Gallimard, [1948], Folio essais, 1985, p. 284.

2 Jean-François Louette, «Sur l'engagement sartrien : les mots », Les Temps modernes, $\mathrm{n}^{\circ}$ 587, mars-avril 1996, p. 71.

3 Jacques Derrida, Poétique et politique du témoignage, L'Herne, Carnets, 2005, p. 9. 
priorités d'ordre affectif devrait, dans une certaine mesure, les expliquer. Il pense aussi peut-être qu'à l'intérieur de cet ordre premier les obligations de la construction syntaxique feront ressortir des rapports de cause à effet. Il y aura cependant des trous dans son récit, des points obscurs, des incohérences même. [...] En fait, au fur et à mesure qu'il écrit son désarroi ne cessera de croître. (G, 310-311).

Lesquels commentaires, on s'en souvient sans doute, valent aussi, ailleurs, pour La Route des Flandres dont la mise en œuvre est cependant bien plus complexe que le témoignage d'Orwell.

La critique simonienne porte d'abord sur la mise en œuvre textuelle du témoignage, du moins ainsi énoncée. Mais elle ne cesse de faire apparaître aussi une autre difficulté. Pouvoir en témoigner suppose une certaine lucidité sur les événements. Jacques Derrida parle à cet égard de "pli de la présence en tant que présence à soi » et il en fait une condition sine qua non du témoignage : «Un témoin ne peut invoquer avoir été présent à ceci ou cela, avoir fait l'épreuve ou l'expérience de ceci ou de cela qu'à la condition d'être et d'avoir été assez présent à lui-même comme tel, à la condition de prétendre en tout cas avoir été assez conscient de lui-même, assez présent à lui-même pour savoir de quoi il parle $»^{1}$. En amont de la refiguration " poétique » ou « poïétique » qui aboutit chez Simon à contester l'entreprise à laquelle elle croyait donner lieu, la pertinence même du témoignage souffre ici d'un autre handicap. L'écriture simonienne ne cesse de dénoncer l'illusion de la présence à soi, au point que j'ai cru pouvoir proposer de lire le prénom " Georges » que le narrateur s'accorde parfois à lui-même, comme signifiant un " je hors-je»: un sujet hors de soi. Simon reproche à Raymond Aron d'écrire une « Histoire coupée de l'expérience sensorielle des faits », mais en fait son grief va plus loin : car l'expérience sensorielle des faits invalide la possibilité même d'écrire l'Histoire. Répondant à Bettina Knapp, qui l'interroge sur la part d'inconscient présente dans ses textes (il y a parfois des questions un peu désarmantes...), Simon répond : «Parler d'inconscient c'est déjà affirmer qu'il se distingue nettement 
du conscient et, en ce qui me concerne, j'ai déjà beaucoup de mal à saisir ce que j'ai vraiment vécu, ce que j'ai cru vivre ou imaginé vivre $\|^{\prime}-$

\section{Comme l'écrit Pierre Bergounioux :}

Ce dont [Simon] est la victime, le témoin, le protagoniste l'affecte sans qu'il puisse en rien dire que : «Bon Dieu !». Il se découvre affecté par l'événement, mais l'événement par sa brutalité irruptive, sa nouveauté stupéfiante, terrifiante échappe à l'acte subjectif par excellence, au pouvoir de nommer qui constitue le monde en objet, le met à distance, le domine. Le style si particulier de Claude Simon se déduit de l'expérience traumatique de sa génération. Ce qu'il en a dit, dans de nombreuses interviews, éclaire assez peu ce qu'il a effectivement fait, la plume à la main, et c'est sans importance. Car c'est un artiste et l'art, ainsi que Durkheim le répète, est « une pratique pure, sans théorie ». L'artiste, l'écrivain n'a pas besoin de savoir. Il lui suffit de faire. La signification de son entreprise se dessinera d'elle-même, sans passer par la conscience claire. ${ }^{2}$

L'auteur de Jusqu'à Faulkner poursuit : «Les écrivains ne savent pas ce qu'ils font. C'est afin de le savoir qu'ils le font. Ils ignorent où mène leur chemin puisqu'ils l'ouvrent, pas à pas, dans leur progression opiniâtre, incertaine, à travers le chaos de l'expérience ». Ce chaos lui-même nuit prodigieusement au témoignage. «Il n'y a plus de masque » dans le témoignage, continue Derrida lors de sa démonstration, formule que nous rapprocherons, a contrano, de la série des masques de boue, de fatigue, des croûtes de crasses et autres écrans qui s'interposent dans l'œuvre de Simon entre le sujet et sa propre expérience. Plus encore, dans le souvenir remémoré et prononcé, les scènes se chevauchent, on le sait bien. Dans Fa Route des Flandres, est-on avec Blum ou Corinne? Qui parle, de Georges ou de Blum? La présence à soi est si vacillante que, dans l'acte même

1 Bettina Knapp, «Interview avec Claude Simon », Kentucky Romance Quaterly, vol. $16, \mathrm{n}^{\circ} 2,1969$.

2 Pierre Bergounioux, «Bon Dieu!», in Cahiers Claude Simon, n², 2006, pp. 36-37. 
du témoigner, le sujet est ailleurs, indisponible au texte de sa propre expérience (au contraire de Rommel, dans LeJardin des Plantes, dont les carnets coïncident avec l'événement, et même avec la maîtrise de l'événement, ce pourquoi, comme le suggère avec pertinence Nathalie Piégay-Gros, ils sont présentés de façon positive par LeJardin des Plantes comme idéal - inaccessible et fallacieux - d'une lucidité de soi à soi). Aussi Simon ne témoigne-t-ilpas ou, pour le dire encore avec Pierre Bergounioux: " C'est en écrivant qu'il pourra se ressaisir, reconnaître le visage obscurci, peut-être absenté, de sa destinée. Ainsi germe la phrase tâtonnante, sinueuse, persévérante qui est la voix même de Claude Simon. Elle est l'effort épuisant, épuisé, invincible pour réannexer ce que l'histoire, dans sa démence, a emporté, à commencer par celui qui écrit, après qu'elle l'a roulé comme fétu dans son cours irrésistible, incompréhensible $\|^{1}$ : et d'inlassablement s'interroger, se réinterroger : " comment savoir ? », " comment étaitce ? », « que savoir ? »...

\section{Configurations textuelles de l'expérience}

L'implication critique de Simon se déploie donc - et donc se mesure - dans un travail poétique de l'écriture. Elle relève non de « discours » au sens philosophique et idéologique du terme - c'est-à-dire d'une quelconque entreprise de systématisation de la pensée -, mais plutôt de ce que Jacques Rancière appelle des «actes esthétiques comme configurations de l'expérience, qui font exister des modes nouveaux du sentir et induisent des formes nouvelles de la subjectivité politique $»^{2}$. Et cette subjectivité est bien politique : elle a quelque chose à dire de lapolis au sein de laquelle l'expérience se configure. S'il fallait inscrire l'activité critique de l'écriture simonienne, ce serait bien en effet du côté d'un "partage du sensible » pour reprendre le titre de Rancière (même s'il peut paraître osé voire incongru de rassembler ainsi Rancière et Foucault autour de Simon). Et encore faut-il s'interroger sur les implications de chacun des deux termes

\footnotetext{
Ibid.,. p. 40.

2 Jacques Rancière, Le Partage du sensible. Esthétique et politique, La Fabrique, 2000, p. 7.
} 
qui composent cette formule quant à l'œuvre de Claude Simon. Je ne le fais pas ici en reprenant les thèses du philosophe, mais en m'attachant pour l'heure à la seule formule que je lui emprunte.

Qu'il y aille du sensible, d'abord, nul doute. On renverra ici à toute la dimension phénoménologique de cette écriture que je n'ai pas besoin de commenter pour des lecteurs attentifs. Toute la charge simonienne repose sur un déni du concept auquel l'œuvre substitue : du côté de la «question primordiale» une matérialité (comme ces « chaussettes et caleçons » que le narrateur de La Route des Flandres réclame à son père offusqué par la destruction de la bibliothèque de Leipzig) sur laquelle je reviendrai ; du côté de l'être au monde une insistance sur la sensation effective, id est sur le «percept» comme antithèse du « concept ». Cela est désormais bien connu.

Mais qu'en est-il du "partage»? Ici les choses sont plus ambivalentes.

Deux éléments postulent effectivement en faveur d'une recherche de «partage». Le fait d'abord que dans les fictions la plupart des discours du narrateur soient diégétiquement adressés (à Blum, à Corinne, au journaliste du Jardin des Liantes). Le fait, ensuite, que dans l'économie narratologique des romans, la postulation d'un narrataire est induite par les formes mêmes de l'expression, qui mime volontiers une certaine oralité, ou un acte de communication (voir par exemple la récurrence des « à supposer que...»).

En revanche, ce « partage » n'institue jamais une « communauté » qu'il contribuerait à fonder en s'adressant à elle. "Aucune communauté ne se dessine à l'horizon du roman de Claude Simon $»^{1}$, souligne avec pertinence Nathalie Piégay-Gros. Dans la série des «témoigner pour» dont Derrida analyse les acceptions possibles dans Poétique etpolitique du témoignage, aucun ne convient à décrire l'écriture simonienne. Simon n'est ni avocat, ni pédagogue, ni tribun : il n'écrit pas en faveur de quelqu'un, ne porte laparole de personne, ne prétend pas éduquerson lecteur et ni s'adresser i quelque force politique potentielle. Force est de constater que le "pour» de Simon demeure sans 
écoute préfigurée par le texte. Même l'entretien avec le journaliste duJardin des 'Plantes est un fiasco. Il ne peut pas comprendre, son Umwelt est trop radicalement étranger à celui de l'expérience rapportée : «Il a dit : Oui, je comprends je... J'ai dit : Non Vous ne comprenez pas. Vous ne pouvez tout simplement pas C'est absolument impossible » (JP, 97 / Pléiade 970).

S'il y a bien, chez Simon, un appel à l'élan de survie, à une sorte de vitalisme animal, jamais cet appel ne configure un groupe ni ne s'adresse à quiconque. Il est plutôt un donné de l'expérience, que partagent Georges dans La Route des Flandres ou O. dans Les Ge'orgiques, réduit pour sa survie au « degré zéro de la pensée» $(\mathrm{G}, 346)$. L'instinct de survie ne se décide pas, il se constate et s'éprouve. Aussi jamais ne se destine-t-il à une " mise en commun », à un "partage $»$, jamais ne se configure-t-il en paradigme qui demanderait à être obéi. Aussi son écriture semble-t-elle relever d'une finalité sans fin, n'ayant d'autre but qu'elle-même.

Chez Simon, la conscience d'appartenir au monde et la critique qui s'élabore sur son état et ses manières ne débouche du reste pas, contrairement à l'appel de Marx dans ses Thèses sur Feuerbach, sur l'effort d'en changer. Non qu'il consente à son ordre, mais il croit vaines les tentatives révolutionnaires et les considère même avec une certaine causticité. Simon conteste la «mythologie héroïque de l'engagement » à laquelle, comme le souligne Benoît Denis, « l'écrivain engagé, quelles que soient sa rigueur et son honnêteté, ne peut éviter de souscrire ${ }^{1}$. Ses critiques et sarcasmes envers les républicains, anarchistes et communistes du Palace le montrent nettement. Ce n'est pas pour autant le signe d'un pur esthétisme, qui se satisferait de ses manipulations et de ses agencements formels.

Car il y a tout de même deux éléments qui contribuent à une forme de partage. Le premier n'est pas loin d'une pratique, préconisée de fait par Sartre, et qui s'accomplit dans le mouvement de l'écriture simonienne : celle du dévoilement. Pour Sartre, l'écrivain est «l'homme qui nomme ce qui n'a pas encore été nommé ou qui n'ose 
pas dire son nom» ${ }^{1}$. Le commentaire que Benoît Denis propose de cette formule décisive de Qu'est-ce que la littérature? vaut aussi bien pour Simon : «l'écrivain est plongé dans la vaste complexité du monde pour en révéler certains aspects ou certaines facettes qui n'avaient pas retenu l'attention jusque là ; la nouveauté de son action ne tient pas au fait qu'il ajoute du neuf à ce qui existait déjà, mais à ce que son intervention porte sur ce qui n'avait pas encore été montré ou dit [...] il modifie donc le donné par le seul effet de sa parole dévoilante $»^{2}$. Ce que ne dément pas le programme du Discours de Stockholm : «Non plus démontrer, donc, mais montrer [...] non plus exprimer mais découvrir. » (DS, 29 / Pléiade 900-901). La phrase de Simon dévoile le dessous des cartes, sonde les reins et les postures, interroge sans relâche et le monde qu'elle décrit et sa façon de le décrire.

Le second élément, chaque lecteur de Simon sans doute en a fait l'expérience : c'est celui de la captation. Le rythme de la phrase, son élan, son allant, ses relances, sa sinuosité, sa réflexion in actu sont tels que le lecteur s'y trouve aspiré, quasiment jusqu'à l'apnée réflexive. La phrase, certes, ne s'adresse guère à lui, ne dessine pas les contours d'un auditoire ni ne fonde une communauté, mais elle refait faire au lecteur l'expérience de sa venue, lui fait éprouver ses doutes et ses corrections, ses agacements et ses analogies. Car c'est une phrase que nul ne saurait maîtriser : il est impossible d'en prendre la mesure, de la surplomber, d'en visualiser d'un seul coup toutes les déterminations, tout l'agencement. Et donc elle impose de consentir à l'immersion, à la déroute logique. Elle met en crise toutes les stabilités intellectuelles sur lesquelles nous fondons nos jugements. Franck Neveu parlait à son endroit d'« agression de la capacité cognitive ». Sa pulsion discursive est disruptive : elle rompt les enchaînements trop logiques de la logique rationnelle pour en découvrir d'autres, plus secrets, plus obscurs. Et faire éprouver au lecteur le dérangement de cette épreuve. Aussi ne construit-elle pas une communauté mais aiguise l'esprit critique sur le plus commun de nos opinions : elle défait les apparences en reliant des pôles apparemment incompatibles.

\footnotetext{
1 Jean-Paul Sartre,Qu'est-ce que la littérature, op. cit. p. 29.

2 Benoît Denis, op. cit., p. 66.
} 


\section{Matérialité et auto-représentation}

J'ai laissé en suspens la question de la matérialité. Elle s'explique bien sûr par la dimension phénoménologique de l'œuvre, et s'inscrit dans un mouvement plus général de la réflexion esthétique du siècle qui permet, notamment, de rapprocher Claude Simon de Francis Ponge. Dans «La Question primordiale», Dällenbach laisse entendre que le passage de Fa Route des Flandres sur la destruction de la bibliothèque de Leipzig condamne à la fois l'humanisme traditionnel, sa croyance dans la Raison et la Vertu, ses « reviviscences » que sont les littératures de l'absurde et de l'engagement - et plus généralement les livres mêmes, en prônant un "retour à la chose ", particulièrement aux choses essentielles que sont chaussettes et caleçons, sucre et saucisson. Mais, cette question associe plus fortement l'œuvre de Simon à des déplacements fondamentaux de l'art après la Seconde Guerre mondiale. Les installations de Beuys, de Kounellis, de Boltanski ou, pour se limiter aux exemples mentionnés par Simon luimême, ceux de Tàpies, de Louise Nevelson, de Rauschenberg... ne peuvent voir leur portée réduite à prolonger le geste d'interrogation de l'art de Duchamp. Ils inventent une autre forme d'interpellation du corps social, après le «trou noir d'Auschwitz et du Goulag»" ${ }^{1}$. Et cette esthétique ne trouve pas sa fin dans le simple refus des discours : elle s'élabore comme une véritable éthique de la matérialité. Les artistes mentionnés n'exhibent pas n'importe quels objets : ce sont des linges maculés, des couvertures militaires, des poutrelles, des rails de chemin de fer, des vêtements entassés, des photographies de journaux, des ardoises brisées... toutes choses qui témoignent de l'Histoire et de ses violences.

Or le seul point de vue tenable sur l'Histoire est bien celui qui passe par la prise en compte, et même par le plus strict respect, de cette matérialité qui excède tout discours. Si l'œuvre de Simon ne cesse de récuser les versions scientifique, épique, littéraire, idéologique et même historique de l'Histoire, il s'arrête en revanche à sa dimension matérielle, qu'il s'agisse de l'expérience sensible - la confrontation, par exemple, à « la matière libérée, sauvage, furieuse, 
indécente (le mélange, la combinaison de quelques poussières inertes, de minerais, de choses extraites de la terre et s'enflammant pour ainsi dire d'elles-mêmes avec cette démesure des éléments naturels, éclatant, se déchaînant par une sorte de revanche sur l'homme, de vengeance aveugle, démente » $(G, 289)$ - ou qu'il s'agisse des documents - cahiers de la vieille Marie dans L'Herbe, cartes postales coloniales dans Histoire, correspondance du Général d'Empire dans Les Géorgiques, archives militaires de Vincennes ou de Rommel dans Le jardin des Plantes - ou encore qu'il s'agisse de « cet inextricable, monotone et énigmatique sillage des désastres » $(R F, 205$ / Pléiade 334) sur lequel insistent La Route des Flandres (voir $R F$, 17, 29 / Pléiade 202, $209 ; G, 362$ ) et même, parfois plus cursivement, les autres romans. Pour rendre compte de cette prégnance concrète du monde, Lucien Dällenbach oppose le matérialisme "à la Lucrèce » de Simon au matérialisme « dialectique $\|^{1}$ du marxisme. C'est-à-dire un matérialisme sans discours téléologique.

Il n'y va pas seulement d'une « homologie structurante entre rupture esthétique et révolution politique $»^{2}$ comme celle que Benoît Denis prête aux avant-gardes, mais d'une pensée concrète du monde et de l'Histoire, et cette pensée concrète, cette concrétion de pensée si l'on veut, excède la simple phénoménologie du sensible pour s'ouvrir à une véritable «morale du corps ». On voit assez bien, je crois, ce qu'il est possible d'entendre dans cette formule que je propose en songeant à l'irrépressible pulsion de survie qui anime plusieurs romans. Mais, au-delà, la formule permet de rendre compte aussi d'une pratique d'écriture fréquente chez Simon. Je voudrais, pour le montrer, opérer un ultime détour.

Dans son livre La Langue est-elle fasciste ? Hélène Merlin-Kajman commente le passage de L'Espèce humaine où Robert Antelme rapporte avoir, lors de son transfert de Gandersheim à Dachau, soutenu la résistance de son corps enchaîné à la déliquescence en recourant à une « image d'esclave à laquelle on est habitué depuis l'école », dont, l'ayant traduite en mots, il se « répét[ait] intérieurement les mêmes lambeaux de phrases, comme un halètement». Voici ce qu'Hélène

2 Benoît Denis, op. cit., p. 24. 
Merlin dit de ce passage : «C'est un lieu commun, une phrase topique, qui le soutient, qui maintient un éthos là où il n'y a plus, comme l'indique Blanchot, de souveraineté subjective. Il permet d'habiter son corps sans s'y engloutir, de transformer l'expérience en scène $»^{1}$. Or nous trouvons quasiment le même récit de la part de Simon, dans un entretien accordé à Jean-Paul Goux pour la Nouvelle critique

«Voyez-vous, quand, prisonnier, exténué, crevant de faim et de soif, je retraversais avec des milliers d'autres la Belgique à pieds, ce qui m'a le plus aidé ça a été de me dire, comme Stendhal au saint Bernard: "Je vois donc une chose difficile !" et plus même : "je vois une chose de tous les temps". Parce que je pensais que le cortège des Juifs emmenés en captivité à Babylone il y a des siècles, eh bien, hormis les costumes et le paysage, ça devait être assez exactement la même chose. [...] On oublie presque (presque : ça ne se laisse jamais oublier) la faim, la soif, la fatigue. Ou du moins on réussit ainsi tant bien que mal à les surmonter, à ne pas s'avilir... $\|^{2}$.

Là, se trouve, me semble-t-il, la clef de la démarche simonienne, dont nous pourrions analyser les éléments :

1. Nécessité de se dire. De se dire soi-même dans la situation, vécue puis mémorisée, et remémorée. C'est-à-dire : non pas seulement vivre l'événement mais, peut-être pour n'en être pas abattu, la nécessité de se voir et de se dire le vivant. Entourer l'événement de phrases, avec une insistance, une pulsion et une pression locutoires qui ne se démentent pas l'événement passé. On a beaucoup glosé autour de la notion de "passé qui ne passe pas », et moi-même aussi, en étudiant notamment le « ressassement» simonien ${ }^{3}$. On a trouvé dans ce passé indépassable la raison de sa répétition dans le texte qui ne cesse de l'inscrire. Plusieurs commentateurs de l'œuvre, en

Hélène Merlin Kajman, La langue est-elle fasciste?, Seuil, 2003, p. 212.

2 Claude Simon, «Un homme traversé par le travail », La Nouvelle Critique, n 105 , juin-juillet 1977.

3 Dominique Viart, «Formes et dynamiques du ressassement : Giacometti, Ponge, Simon, Bergounioux », revue Modernités, $\mathrm{n}^{\circ}$ 15, 2001, pp. 59-74. 
réfléchissant à ce que j'ai appelé la « poétique de l'épanorthose » ${ }^{1}$, ou d'autres à la notion de répétition (Stéphanie Orace, Claire Guizard) et de reformulation (Stéphane Bikialo), ont aussi beaucoup commenté ces pratiques d'écriture comme marques d'une insuffisance du langage que l'œuvre s'évertuait à la fois à dénoncer et à réparer. Mais ce que nous avons manqué jusqu'à présent, me semble-t-il, c'est que la nécessité de parler - de se parler, intérieurement, puis de parler à (parler à Blum, à Corinne, ultimement au lecteur) - s'originait dans un tel effort pour ne pas succomber, dans la nécessité de recouvrir la souffrance subie de langage pour ne pas s'y engloutir, de recourir à l'expérience du langage pour ne pas succomber à celle de l'Histoire. Se voir vivre les choses - et se dire intérieurement ce que l'on vit -, c'est déjà installer une distance entre soi-même et sa propre souffrance. L'œuvre tout entière, finalement, ne fait que prolonger ce qui a commencé au moment de l'expérience elle-même. Et qui s'y est noué en tant que modalité de survie. Un tel processus n'est pas sans rapport avec l'économie particulière de la «mélancolie» selon Simon. Si, comme l'explique fortement Le jardin des Plantes, celle-ci n'est pas une complaisance romantico-symboliste à son propre alanguissement, mais, au contraire, la rage de survivre, et de retenir tout un monde présent sur le point de disparaître parce qu'on disparaitrait au monde, alors la pulsion verbale est bien de même nature. C'est une survie enragée : une leçon de résistance.

2. La transformation de l'expérience en scène, selon la formule d'Hélène Merlin, sa visualisation analogique et décalée, permet aussi de comprendre un second élément insistant de l'œuvre, une autre de ses pratiques récurrentes : le recours à toutes les comparaisons théâtrales ou cinématographiques, auquel nous pourrions, du reste, ajouter la quasi totalité du système hypothético-comparatif régi par les « comme si » qui scandent et relancent les phrases simoniennes. Il s'agit là du besoin de se représenter, de rattacher les choses perçues aux topiques qui permettent de les recevoir. La misère, " quasi deshistoricisée, est immémoriale, archaïque $»^{2}$, écrit Nathalie PiégayGros, qui fait de cette déshistoricisation la preuve d'un dégagement

1 Dominique Viart, Une mémoire inquiète, PUF, 1997.

2 Nathalie Piégay-Gros, art. cit., p. 285. 
de l'Histoire. Il me semble plutôt qu'il s'agit de s'accorder les moyens de vivre l'Histoire - ou d'y survivre.

\section{Vers les « fictions critiques»}

On aura compris au fil de ce parcours que l'écriture simonienne ne peut obéir aux injonctions sartriennes d'engagement de la littérature. Mais elle ne le peut parce qu'elle n'est pas en situation de le faire. Dire les choses ainsi est une façon de ne pas trancher radicalement le lien de pensée entre les deux écrivains. D'autant que l'écriture de Simon ne renonce pas à l'immersion ni au dévoilement, quand bien même ce dévoilement serait toujours à réinterroger, jamais donné une fois pour toute. "Critiquer» signifie mettre en crise, et non rechercher ni, a fortiori, affirmer un principe de cohérence. Au contraire : dans la critique simonienne, c'est la cohérence qui est suspecte. Suspecte d'avoir été surajoutée au monde et d'en fausser la réalité. Aussi l'œuvre de Simon est-elle bien critique, et substantiellement. Elle l'est d'un monde et de ses valeurs (L'Acacia, La Route des Flandres, Histoire en administrent la leçon), y compris de ses illusions (Le Palace) ; elle est critique, au-delà, envers les discours qui recouvrent le monde et qu'il est nécessaire de déchirer pour l'entrevoir autrement. Tout cela relève, in fine, d'une critique épistémologique et, à certains égards, philosophique. Cette écriture est critique, enfin, de ses propres procédures (et plus encore de celles mises en œuvre par la littérature). Ce faisant, elle ouvre la voie à bien des pratiques qui se sont déployées depuis, dans la littérature contemporaine, du côté de ce que j'ai proposé d'appeler des «fictions critiques $»^{1}$. À cet égard, nul doute que Claude Simon soit l'un des précurseurs majeur de ces nouvelles formes littéraires. La différences avec Sartre est qu'il n'a jamais produit de discours théorique autour de cette pratique, et que c'est sa pratique, sa phrase, qui ont suscité l'émergence d'une autre façon d'engager l'écriture dans la critique du monde ${ }^{2}$.

\footnotetext{
1 Dominique Viart et Bruno Vercier, La Littérature française au présent, Bordas, 2005.

2 À propos de cette influence de la phrase simonienne sur les écrivains de la génération suivante, on se reportera utilement aux contributions du volume $n^{\circ} 2$ des Cahiers Claude Simon.
} 